\title{
Variations
}

Variations

Revue internationale de théorie critique

$21 \mid 2018$

L'industrie de la culture : version originale

\section{Industries musicales: Créateurs, artistes ventouses et production du public}

\section{Bruno Lefebvre}

\section{OpenEdition}

\section{Journals}

Édition électronique

URL : http://journals.openedition.org/variations/911

DOI : 10.4000/variations.911

ISSN : 1968-3960

Éditeur

Les amis de Variations

Référence électronique

Bruno Lefebvre, «Industries musicales: Créateurs, artistes ventouses et production du public ", Variations [En ligne], 21 | 2018, mis en ligne le 05 avril 2018, consulté le 03 mai 2019. URL : http:// journals.openedition.org/variations/911 ; DOI : 10.4000/variations.911

Ce document a été généré automatiquement le 3 mai 2019.

Les ami•e•s de Variations 


\title{
Industries musicales: Créateurs, artistes ventouses et production $d u$ public
}

\author{
Bruno Lefebvre
}

«Seul le poisson mort suit le courant »

(proverbe, prétendument chinois)

\section{Introduction}

1 La production musicale peut s'aborder selon le modèle socio-économique des «entreprises en réseau». Pour saisir cette forme mouvante, j'ai mené une série d'entretiens individuels ou collectifs avec des musiciens professionnels, quel que soit le «style»: musiques contemporaines, jazz, classiques, ethniques, variétés, etc..., indépendamment de tout jugement esthétique ou moral. Je procède par "grappes " sociales centrées autour de mes connaissances, les copain(e)s de copain(e)s. Le présent article cherche à saisir, grâce aux parcours témoignés, quels sont les modes d'apprentissage techniques, les moments décisifs, les institutions, les deals économiques et juridiques (statuts, droits d'auteurs, modes de rétributions) ; quelles sont les marges de choix et de risque pour tracer sa route, comment les spécialités professionnelles périphériques et les partenaires sous-traités, lumières, sons, juristes, agents, " tourneurs ", metteurs en scène, conseillers en tout genre, orientent les trajectoires, la renommée et l'identité de soi. Je remercie de tout coeur les témoignages de Marc Bahuaud, Laurent Berthomier, Olivier Congar, Ismet Emered, Michel Frémont, Daniel Givonne, Hassen Gammaz, Dominique Huchet, Henrick Honstter, Jean Pierre Jagot, Gustavo Ovales, Victor Racoin, François Ripoche, Richard Roy, Michel Perez, Daniel Roux, Tchavolo Schmidt.

2 Les grandes industries architecturales et picturales nous sont décrites depuis bien longtemps, avec à leur tête un savant-artiste donneur d'ordre et ses sous-traités; en musique, il est probable que les grands ou petits orchestres de l'antiquité (Abyssinie, 
Égypte, 4000 ans av JC, le début des industries privées avec la monnaie frappée qui permet de s'émanciper de famille et de la protection alimentaire et résidentielle du dominant) aient fonctionné selon la même organisation. Il faut séduire le Prince ou le Peuple. A partir de la renaissance occidentale et au travers des écrits méthodologiques, muséologiques, religieux, commerciaux, jusqu'aux traces informatiques, électroniques, technologiques d'aujourd'hui, nous constatons qu'il existe sans cesse dans ces industries, le projet d'allier le Prince, nécessairement de bon goût et le Peuple inventif.

3 Les exemples récents sont nombreux, depuis les Hongrois qui employaient au XVIII ${ }^{\circ}$ siècle des Tziganes, terme synonyme d'esclave chez les Manouches, pour composer des opéras (d'où les gammes "hongroises", exception au code classique) en passant par Ravel et Debussy à la recherche de thèmes populaires hispanisants ou Cubains, jusqu'à Mick Jagger des Rolling Stones qui demandait à ses bassistes de ne pas jouer plus de trois notes par mesure afin que la musique soit facilement reproductible par les adolescents qui achetaient des guitares et des produits dérivés; le mouvement social, économique, politique est le même, la musique doit fédérer. D'ailleurs, elle adoucit les mœurs, dit-on.

Les nouvelles techniques, radio, télévision, cinéma s'adressant à un public de masse aux USA et en Europe avec Gustave Le Bon et Hermann Goëring ${ }^{1}$ et bien d'autres reconfigurent l'art ou les manières de faire de la scène vivante: en Amérique ou en Europe (l'Allemagne et les USA sont les premiers producteurs mondiaux de films de romance à l'époque), les « vedettes » de l'époque, ou «têtes de gondole » comme on dit aujourd'hui péjorativement dans les industries de la diffusion, apprennent aux musiciens les mimiques et techniques du corps, comme chez les acteurs de théatre, comment bien se comporter sur une scène ou à la TV à partir du moment où le cinéma est parlant. Depuis les années 80, les gamins de 6 ans savent répéter et mimer parfaitement les dessins animés des Mangas japonaises, «La force est avec toi !», mais il est touchant de revoir nos artistes chanteurs francophones maladroits des années 50, puis ceux qui sont les "enfants de la télévision » et enfin ceux qui, malgré la beauté de leurs chansons, sont oubliés. Les spectateurs font le tri et maintenant avec les réseaux sociaux, tout le monde peut se mettre en spectacle, avec plus ou moins de bonheur, mais le musicien qui n'aurait pas un minimum de " présence » sur scène, en terme de gestuelle, sourire, habillement, intonations, bons mots, se ferait immédiatement rejeter. Des émissions de pseudoconcours de nouveaux artistes (que tout le monde dans le milieu connait parfaitement par ailleurs) à la TV formatent les goûts et pulsions d'achat des consommateurs qui votent et l'industrie est là pour les servir.

\section{Le laboratoire de la flexibilité}

Pendants les années 1960, les entreprises de transport routier incitaient les chauffeurs à se mettre à leur compte, devenir indépendant en leur proposant de leur prêter de quoi acheter un tracteur, suivi vers 1990 par les industries classiques qui incitaient ouvriers et techniciens - les secrétaires et comptables étaient déjà rompus à ce genre d'exercice - à se mettre à leur compte en prévision des libres échanges qui se profilaient dans la future Constitution Européenne. Le statut des intermittents du spectacle, créé en 1936 pour avoir une main d'oeuvre fidèle et bien formée dans les domaines stratégiques de la radio et du cinéma au début des guerres européennes se complète en 1939 avec les congés payés et les assurances chômage en 58 , les artistes-interprète et les techniciens du spectacle vivant sont intégrés (comme les caméramans des matchs de football) en 1969. 
6 En ce sens, les intermittents sont les précurseurs des formes d'organisations flexibles et branchées, modèles des styles de comportements socialement valorisés, que ce soit le travailleur en artiste $^{2}$ ou l'artiste en travailleur ${ }^{3}$. Selon les idéologies de la sociologie américaine, il s'agit d'être « entrepreneur de soi- même », le nombre de CDI ne représente pas la moitié des emplois, il faut incorporer le modèle «japonais» ou tout autre dénomination exotique, « créer » son poste de travail, accepter les liens de subordination et les contrôles hiérarchiques entre intermittents, intérimaires, sous-traitants, bénévoles, prestataires, coachs, selon les types de contrats, et sont appelés à faire preuve d'une autonomie responsabilisante. Les prises de risque qui n'ont pas forcément un résultat positif sont appréciées, la gestion de l'incertitude doit permettre une expertise technique, financière et relationnelle auto-contrôlée, Il faut montrer que l'on fait des efforts, être souriant, sympathique, disponible sexuellement et si possible, pas trop demeuré. Ainsi intègre-t-on une niche de spécialisation productrice d'innovations selon les définitions des sciences et techniques.

7 Les free-lance et nombre d'entreprises artisanales produisent donc une "perfection concurrentielle ", l'employeur ou le commanditaire n'est pas responsable de la carrière des prestations multi-salariales et le chômage indemnisé sous contrôles des syndicats permet une externalisation du coût des maintiens des stocks des ressources humaines. A un montant de prestation fixe s'ajoute une variable individualisée et éventuellement un intéressement au résultat car il faut conserver le vivier. Combien d'ex-vedettes ou de groupes effectuent un « retour » réussi après des années d'absence !

8 Donc, le consommateur nostalgique devient acteur, on ré-écoute Tino Rossi, Dalida et Claude François, les firmes deviennent créatrices et les injonctions médiatiques suscitent un engagement productif de ressources personnelles et collectives, les produits deviennent des «styles" musicaux dont les dénominations des mêmes musiques changent au fil du temps : qu'est ce qui est blues, jazz, africain, négro-latino, world ou encore qui s'inspire de qui entre le music-hall et les «créateurs $»^{4}$ puisque les musiciens et compositeurs passent facilement, par contrat, de l'une à l'autre?

\section{Les modèles de ventes}

9 L'argument de vente principal pour la musique comme toute autre marchandise est la nouveauté dans le son, la composition, l'instrumentation, il s'agit d'innovations incrémentales qui supposent l'accumulation et la maîtrise des techniques pour réorganiser les savoir faire. L'innovation radicale est beaucoup plus rare, on pourrait citer par exemple l'utilisation de l'informatique et de l'électronique par le groupe Kraftwerk en 1970 qui va influencer Pink Floyd ou Soft Machine puis se retrouver au niveau technique dans tous les studios d'enregistrement disco, funcky puis techno. Les tribulations des innovations techniques ${ }^{5}$ nous montre que d'un point de vue historique, il y a peu d'oublis ou de déchets car les simulacres réempruntent sans cesse de vielles recettes. Il n'y a jamais abolition des savoir faire anciens. Des chanteurs du show-business ont bâti toute leur carrière sur un seul titre diffusé régulièrement dans les boites de nuit.

Les logiciels de pointes, ou " high-tech », de calcul aléatoire utilisés par les opérateurs en Bourse qui intéressent la classe bourgeoise vont donc se retrouver dans l'informatique musicale. Mais les experts juridiques et les gestionnaires devront alors mettre en place des contrôles décentralisés et des auto-disciplines, des divisions sophistiquées du travail. 
Le monde des Arts qui exhibe le luxe et qui produit des profits économiques et politiques doit trouver une figure séductrice pour les masses de consommateurs et l'on choisit la tête de scène, chanteur, chef d'orchestre, musicien qui correspond le plus au mythe de l'artiste du XIXème siècle: frivole, capricieux, exigeant et joueur, incompris selon le modèle du garbage can dans les typologies sociologiques des organisations d'entreprises américaines, mais sensible et techniquement compétent.

11 L'idéalisme sacrificiel de l'artiste du XIXème siècle qui fait don de lui-même et s'oppose au matérialisme calculateur bourgeois va donc se décliner selon les normes de validations et les arrangements locaux et conjoncturels, ce qui génère de multiples variantes. Les industries de la critique sociale des années 1967/75 sont passées de mode, comme le protest song, remplacées par l'écologisme, ce qui permet à la jeunesse de se détourner de la fâcheuse idée de lutte des classes; on retrouve également ce phénomène dans les arts plastiques contemporains lorsqu'on lit les journaux et les fascicules d'exposition, quelque soit le pays d'Europe. Les mouvements de retour à la terre qui ont allié un temps les populations rurales traditionnelles, les catholiques sociaux et les jeunes urbains diplômés précaires comme dans le Larzac en 1971 sont typiques de cette matrice qui va générer des revendications localistes ou nationalistes comme dans le rock anglais.

12 Ce qui se vend le mieux dans tous les pays sont les chansons d'amour, évidemment, mais aussi le localisme ou le nationalisme peuvent être érigés comme des marchandises et des politiques culturelles comme arme, sur le modèle d'une contre-culture résistante ou exploitée : nous connaissons tous le Raggae de Jamaïque, alors que lorsque je m'y suis promené, je n'y ai entendu (avec plaisir) que du Calypso des Caraïbes; la formidable politique d'éducation scolaire des jeunes à Cuba, (loin devant les performances des jeunes français du même âge en écriture et calcul) selon laquelle « il faut danser et jouer pour la révolution ", exporter des artistes internationaux et faire rentrer des devises (de la même manière que Cuba exporte ses médecins dans toute l'Amérique du Sud); ou plus récemment, l'engouement pour les styles "root-Africa", élaborés par les studios d'enregistrement belges mettant en contact les Cubains et les Africains dans les années 50 (selon l'exposition Royale de Belgique en 2004, Bruxelles). Que ces musiques et politiques d'exportations culturelles de la part de pays pauvres soient copiées, pourquoi pas? Les puristes pourront toujours essayer de mettre en scène l'original.

Les modèles et les contre-modèles font donc union et produisent de nouveaux territoires comme Ibiza ${ }^{6}$ autrefois ou maintenant plus branchés, l'Indonésie, où l'hédonisme touristique, les sauna, les massages, les vacances, alliés à de la musique branchée (comme Jimmy Hendrix est venu passer une semaine de vacance à Majorque en 1970) ou de la techno soft large public qui font venir d'abord des gens aisés puis les low cost et les clubbers. L'ensemble nous donne une joyeuse idéologie où le temps et l'argent n'existent pas (un peu comme dans l'imaginaire de certains socialistes), où il n'y a plus de différence entre travail salarié et temps libres, où l'on accède à l'autonomie sans passer par la formation, où l'on a conscience de soi-même et donc on se réalise en sautant sur place au rythme des tempos et boites à rythme. Mais la protestation contre le capital et les conditions de vie se vendent parfois très bien aussi : les rebelles à la domestication des marchandises musicales, les pirates, les classes d'âge jeunes opposées à la gérontocratie trouvent des alliés : l'articulation entre les bobos écolos et les punks s'opère très bien, comme dans quelques quartiers cosmopolites de Berlin Est.

Puisque le modèle de vente est la mise en scène d'un principe d'innovation, il faut donc spéculer sur les talents, organiser des principes de justification qui permettrons des 
formes d'exploitation acceptables au travers d'organisations et de conventions flexibles selon les besoins. Les concours, prix, diplômes, distinctions, les labellisations, évaluations, formalisations, exclusions, n'engagent que ceux qui sont payés pour les réaliser. Pour stabiliser les incertitudes du marché musical, les institutions de la reproduction des savoir faire sont en France les écoles, université, instituts, fédérations d'artisans et syndicats, tous tendus vers la reconnaissance de l'État et des Directions Régionales de l'Action Culturelle, à l'origine le projet était ambitieux et généreux, il y avait de l'argent pendant les années 80 (j'avais tenté de m'y placer en Rhône Alpes, mais j'étais «trop chercheur » et pas assez administratif ; reproche fréquent).

L'économie des talents est difficilement quantifiable selon les histoires locales et les prix, mais on peut repérer quelques inventions surprenantes, comme celle de la tulipe des horticulteurs des Pays Bas au $\mathrm{XV}^{\circ}$ siècle, remplacée depuis par une plante devenue idiote, l'orchidée séparée de son milieu naturel. Le talent est sujet à de multiples différentiations, illimité selon des critères peu stables, une seule chose est sûre selon les mythologies de la parenté : il s'hérite. Les fils et filles, voire même les arrières petits neveux d'un tel ou une telle sont connus dans les domaines du cinéma, de la télévision, de la musique et ailleurs bien sûr. Mais l'hypothèse est hardie, ne s'agirait-il pas de réseaux sociaux et de capital de classe comme disait l'un de nos illustre prédécesseur?

Le travail critique des catégories initié par les sciences, et pas seulement nationalesociales, nous oblige à relativiser ces histoires de talents : Au Portugal, la musique est enseignée dans les écoles polytechniques, puisque la musique est une technique qui consiste à faire vibrer des lamelles d'air. Pour poursuivre dans ce genre d'idées bassement matérialistes, nous avons en occident 12 gammes dont deux mineures (pas de quoi fouetter un chat) plus quelques autres « dissidentes ", hongroises, napolitaines, flamenca, en Iran 53 gammes, en Inde 57 gammes. Où est le talent et le «progrès »? Ces choses-là ne sont sûrement pas cumulatives, pas plus que les connaissances en sociologie ou en créativité, en culture ou en littérature.

Il n'en reste pas moins que les industries musicales, au travers des prix, des cachets, des hit-parades, des auto-célébrations vont « développer » un artiste (de variété par exemple) de la même manière qu'on développe un poulet ou un poisson en élevage. Celui-ci dira en interviews publiés combien il lui a fallu de courage pour vaincre les adversités, de ténacité, de travail, un peu sur le modèle des récits de vie et des mythologies «percevaliennes» selon Claude Lévy Strauss ${ }^{7}$, il faut "en vouloir» pour devenir un héros, et ensuite " winners take all markets! ». Très souvent, la carrière professionnelle est mise en scène comme un jeu de hazard où des «bonnes fées", des producteurs ou des leviers relationnels se seraient penchés sur le génie débutant qui grâce à son endurance, son mérite, après des moments de galère et une basse extraction sociale, se serait intégré au milieu du business. Selon ces idéologies individualistes qui ne viennent pas forcément de la réforme protestante (Max Weber) mais aussi de l'emprunt à des codes aristocratiques anciens ${ }^{8}$, les «gros lots » sont réputés, les municipalités lorsqu'elles ont une urgence électorale invitent des « stars ", tout comme les candidats à la présidence de la République (et certaines s'y sont grillé les ailes, désavouées par leur propre public). Dans cette ambiance individualiste du milieu musical, il semblerait que les très grands écarts de revenus soient souhaités selon certains sondages (mais être guitariste ou bassiste un moment pour Johnny Hallyday n'amène pas d'argent direct, simplement une ligne dans un $\mathrm{CV}$, donc négociable plus tard), le rendement des talents est donc démultiplié par les médias et la jet-set. Collectionner les talents pour certains 
producteurs est inséparable d'organiser la concurrences entre eux (ce qui peut provoquer de la souffrance et des suicides comme dans n'importe quelle autre organisation du travail), même si rechercher des talents, tâche plus noble, suppose investir, les former, créer leur style, ce qui nécessite une réquisition physique et cognitive importante.

Depuis 1987, la quantité de réalisations reste constante en France, le nombre de jour travaillé par les intermittents a baissé, tout comme la durée des contrats, mais leur nombre a augmenté et les effectifs des musiciens déclarés a été multiplié par deux ou deux et demi. Selon ce joli modèle libéral, la productivité augmente avec la mise en concurrence des travailleurs.

\section{La production institutionnelle des artistes en France}

19 Selon les vieux réflexes franco-jacobin, «l'Etat peut payer », « l'Etat peut investir »; cette révélation de la mentalité collective est bien différente que celles initiées par la United States International Agency, le British Consil ou le Goethe Institut. Les ONG disposent également d'une très forte autonomie pour produire des artistes de manière internationale. Les industries de la culture génèrent un chiffre d'affaire de 62 milliards d'Euros chaque année, (autant que les impôts directs ou autant que les taxes sur l'essence) et alliées avec les industries du tourisme, elles apparaissaient comme une alternative facile aux yeux des élus et gestionnaires des territoires pour remplacer les emplois dans les sites industriels en voie de disparition, les exemples sont nombreux. La promotion des festivals, de la beauté des villes, de la qualité de leurs services doivent pallier à la dés-industrialisation où l'on remplacerait les salaires fixes des ouvriers et techniciens par les revenus faibles et fluctuants des livreurs de pizza à domicile de statut uber, où les moules-frites, d'un meilleurs rapport, remplaceraient le steack-frites. Malgré l'invention d'un folklore, de spécialités culinaires et produits authentiques, de la publicité des paysages fantastiques, de la mobilisation des syndicats du commerce et des hôtels restaurants, des centaines de festivals disparaissent chaque année, comme le montrent les cartes : les municipalités n'ont plus de finance.

L'intervention de l'Etat dans les domaines des industries culturelles est une vielle tradition française depuis Colbert en 1664 avec l'intendance générale des bâtiments du Roi jusqu'à «l'exception culturelle » de J. Lang pour protéger les industries du cinéma français de la concurrence étrangère il y a quelques années. Cette longue tradition permet à l'Etat de capter les disciplines, les institutions et de fixer les normes culturelles par la production d'académie au fur et à mesure que la censure de l'Eglise se laïcise. Le patronage monarchique et les logiques de mécénat sont centralisés de manière nationale, comme les Archives, la Bibliothèque, le Museum central des Arts, les Monuments historiques, les Arts et Métiers, les Beaux Arts en 1940. Vers 1875, l'Art Républicain se doit d'assurer l'enseignement, les besoins généraux, d'inculquer le sentiment de l'amour du beau, de consacrer le génie national, de sélectionner les élites, en 1902, avec la création juridique de la propriété intellectuelle, du droit d'auteur et le statut d'entrepreneur, l'Etat devient client et non plus mécène, en 1906, avec la suppression de la censure au théâtre, la reconnaissance des artistes passe par le marché. Les soutiens à la création passent par une inscription sur des listes et des commandes sur programme dont l'exécution est suivie par un inspecteur, que ce soit pour la réalisation des monuments aux morts en 1920 ou pour les manifestations supervisées par les DRAC aujourd'hui. Nous observons que de nombreux artistes ayant chanté le localisme se reconvertissent comme élu à la culture 
dans les municipalités. L'Etat assure encore en partie les aides sociales et la consécration, mais les initiatives privées du divertissement et du Music Hall à partir des années 20, les revues des orchestres et danseuses du Moulin Rouge, l'influence des vedettes internationales, poètes, auteurs littéraires, peintres, les mouvements artistiques, les privatisations de la télévision, le mouvement Yéyé des années 60, vont contrecarrer le monopole de l'Etat .

Les expositions universelles de Paris au XIXème siècle, la création du salon des refusés en 1863 puis celle de la société des artistes en 1884 avait déjà écorné ce monopole, l'art se commercialise et l'innovation devient un critère de réussite, même si l'opéra et les arts lyriques assurent le "rayonnement de la France». Les municipalités prennent de l'importance dans la production musicale et bientôt, le Front populaire (1920/36) promeut une nouvelle génération de militants culturels, le travail administratif de l'Etat, le parti communiste qui veut diffuser la culture savante comme un héritage national, la mise en place des maisons du peuple, des foyers Léo Lagrange, les auberges de jeunesse, les revendications des associations, groupements professionnels et syndicats font accéder les populations aux loisirs. Les activités artistiques se diffusent pendant les camps de vacances, les colonies, les congés payés. Les clubs de peinture et de musique les multiplient autour des entreprises. Le théâtre, la radio sont subventionnés, des aides à la création s'instaurent. "Egalité et intervention de l'Etat» vont devenir une vision cohérente jusqu'en 1970 environ. Les politiques culturelles vont à nouveau se centraliser avec le gouvernement de Philippe Pétain et celui d'André Malraux au début de la V ${ }^{\circ}$ République. Le Ministère des Affaires Culturelle est séparé de celui de l'Education Nationale. On veut faire quelque chose de grandiose tout en continuant de penser le projet du Front Populaire où « la Culture sera gratuite ».

Les Maisons de la Culture abritent des artistes de tous secteurs, Chagal, Miro, Boulez sont promus, le Centre Beaubourg construit et les budgets des Arts et du cinéma sont divisés en deux (400 millions en 1957, 200 en 1967). Les industriels cotisent de moins en moins, "Jeunesse et sport» décline comme la fréquentation des bibliothèques, cette administration sera supprimée, on s'intéresse aux monuments historiques, une politique du patrimoine met en place les enseignements d'ethnologie de la France. Les musées d'Art et Tradition Populaire puis la Mission du Patrimoine Ethnologique contribuent à l'invention d'un folklore local où se reconnaissent les populations et celui-ci entame une belle carrière. Les maisons de la culture deviennent Centres d'Animation Culturelle, les élus conservateurs sont méfiants, les classes moyennes consomment énormément, le projet de démocratie culturelle des mouvements de gauche décline. Différentes villes acquièrent un cachet touristique via des festivals en tout genre.

Mais dans les villes, des centaines d'initiatives associatives construisent des écoles de musique, comme l'AIMRA à Lyon ou le Groupement de Recherches Interdisciplinaires sur la Musique (né en 1982, dont j'ai fait parti). Nous étions une douzaine de formation sociologique et avions six salles de concert qui fonctionnaient en trois/huit heures/jour, la gestion de nombreux contrats de sociologues sur les radios libres, toutes récentes, la demande de salles de concert nouvelles de la part des citoyens et notre budget allait très fort, jusqu'à ce que le fisc nous oblige, avec la complicité des Universitaires selon lesquels la Culture était leur chasse gardée, à déclarer une SARL, devenue une école privée par la suite de formation des techniciens son et lumière. La demande était importante pour des loisirs musicaux, l'Etat construit des conservatoires nationaux de Région, des Fonds d'Intervention Culturelle, des chartes sont signées, les militants syndicaux ou municipaux 
se fondent dans le « Tout Etat Culturel » de Jack Lang en 1981/95. Le Patrimoine devient le jazz-rock, la BD, la mode, la gastronomie, puis le rap et le tag.

Cette ouverture de spécialités authentifiées dans le champ du divertissement développe une logique de l'audimat, les fêtes de la musique sont devenues incontournables et imitées soit disant par nos voisins européens. Mais si l'on compare avec nos voisins européens, les pratiques musicales et les politiques culturelles empruntent bien d'autres chemins. En Grande Bretagne, les supporteurs de matchs de foot, hooligans, chantent à trois voix et en canon, à la différence des Français. A Setubal, au Portugal, des usines en friches sont devenues des sortes de Maison du Peuple où sont présents : les syndicats, la Municipalité, la Maison des chômeurs, un dispensaire médical et bien sûr des salles d'exposition, de conférence, de concerts pour les groupes et les concours d'improvisation poétique de type fado, ce qui romps avec les manies de la catégorisation et ségrégation française ; je trouve astucieux que les chômeurs aient accès à toutes ces institutions et ces services.

En France, les critiques de droite évoquent une «défaite de la pensée » (Finkelkraut), l'Etat deviendrait le pourvoyeur de la consommation de masse. Après «l'exception culturelle » de 1993 pour le monde du cinéma si proche du personnel politique et la «fracture sociale» de 1995, des artistes soutiennent les sans-papiers et demandent des subventions (!). La population des intermittents entre régulièrement en crise à partir des années 2000, le budget de la culture baisse régulièrement, la consommation également, sauf dans le domaine de la musique. De gigantesques usines de spectacle vivant se sont constituées, réquisitionnant les stades, employant par sous-traitance des centaines d'entreprises et professions indépendantes, des convois de 15 à 25 poids lourds, plus des manutentionnaires, électriciens, informaticiens, employés des services d'ordre, et les personnages les plus connus sont les artistes-interprètes, les musiciens, les auteurscompositeurs, les managers, tous considérés comme bienfaiteurs. Les emplois à caractère culturels recouvrent des doubles activités à $37 \%$ en 1991, le nombre d'intermittents passe de 57000 à 125000 en 2004, le nombre de CDI est de 26\%.

Pour autant, les vedettes du show-buisiness, modèles de réussite chacun dans leur style, restent originaires de classe sociales aisées : enfants de la bourgeoisie coloniale (Dalida, Claude François); enfants d'ingénieur ou ingénieur eux même (Boris Vian, Joe Dassin, Henri Salvador, Véronique Sanson, Lavilliers, Antoine) ; enfants du milieu des comédiensréalisateurs (Johnny Halliday et des centaines d'autres), certains ont été initiés au film publicitaire et au théâtre pendant leur prime enfance (Catherine Ringer). Les auteursproducteurs sont devenus gentils et connus (Didier Barbelivien, Michel Berger, JeanJacques Goldmann), des héros pour les artistes et le public. Qu'ils adoptent des postures de rebelle, d'amoureux ou de plaisantin, il s'agit d'épater le public, de produire de la rationalité affectuelle au sens de $\mathrm{M}$. Weber. Les textes et les harmonies doivent être simples pour être répétées par les foules, être populaires.

\section{Le marché du travail}

Les croissances permanentes des coûts des spectacles obligent à des recherches spécialisées pour l'obtention de financements disponibles et des juristes se spécialisent. L'environnement des musiciens est désormais composé d'ingénieurs hautement qualifiés par les écoles ou les Universités. Les nouvelles technologies de l'informatique et électronique permettent une réduction des coûts de production, des associations de 
production de festival s'éloignent des municipalités, après la musique techno et les musiques actuelles, dès qu'ils le peuvent. Les divisions du travail et des revenus se complexifient : comment celui ou celle qui pianote un ordinateur sur scène serait-il plus connu ou mieux payé que le technicien du son qui pianote son ordinateur un peu plus loin afin que le public soit content? Des orchestres d'une quinzaine de musiciens afroaméricains qui ont écumé tous les casinos de la façade Atlantique et de Méditerranée ont été licenciés, le chef d'orchestre est devenu DJ, accompagné d'une chanteuse.

Parmi nos interlocuteurs, tout le monde a donné des cours de musique, que ce soit au noir ou dans des associations pour les plus démunis d'entre eux, ou dans les écoles municipales pour ceux qui sont devenus titulaires d'un diplôme d'Etat dont le concours est devenu de plus en plus théorique, ou au conservatoire. Ceux qui ont une carrière internationale ont fondé des écoles en France ou à l'étranger lorsqu'ils jouent de la musique ethnique, parfois des Master-classes en Suisse pour de la musique spécialisée. Ces derniers avaient des parents musiciens ou mélomanes et ont joué devant le cercle familial dès qu'ils étaient enfant avant d'être encouragé à travailler un ou plusieurs instruments. D'un autre côté, les fanfares, les orchestres connus, rassemblent régionalement des musiciens de toute trajectoire et de tout style lors de fêtes privées, tout le monde a joué avec tout le monde.

Les relations avec les espaces où l'on peut se produire sont de plusieurs types: l'intermédiaire de la Fondation de France dont le rôle diplomatique pour la valorisation de la Culture nationale assure les circuits pour la musique classique, il y a aussi les agents de production privés intercontinentaux pour la musique "non-sérieuse », dans d'autres style, et tout cela occupe une grande partie du temps pour les prospecteurs, tout comme la fréquentation des café-restaurants montés en association de diffusion de spectacles. Il faut aussi rendre visite aux promoteurs et aux responsables de festivals. Les concerts au Japon ou en Corée sont les plus prisés, pour les cachets, l'accueil et la technicité des personnels. Les musiciens travaillent beaucoup. Mon ami Gustavo Ovales, percussionniste vénézuélien international a un emploi du temps réglé : trois heures de musculation et de footing, trois heures de recherche informatique pour trouver des opportunités chez les associations de soutien ethnique et les municipalités, constituer des formations pour telle ou telle occasion, plus $3 \mathrm{~h}$ de répétition et $3 \mathrm{~h}$ de concert local chaque jour. Il possède ses propres agents, auxquels s'ajoutent les agents continentaux d'une grande vedette des Caraïbes, «je joue pour un musicien ».

Mais les revenus ne sont pas énormes et ils ont énormément baissé. Je me souviens d'un vieux Monsieur qui était batteur dans des orchestres yéyé en 1960, il gagnait 10 smic par mois. Les déplacements incessants rompent les couples et les relations avec les enfants, d'autant plus que les métiers de la scène "attirent les filles ». Les doubles activités sont ordinaires. Liens avec l'info-communication, les entreprises de video, de décorations techniques sont fermement établies et composer des musiques de film est d'un bon rapport.

31 De même que les musiciens précaires, sans identification professionnelle, proche du RSA, les intermittents qui ne peuvent pas assurer leur nombre de dates minimales par an et qui risquent de se faire radier de la sécurité sociale prospectent partout et les concurrences sont vives, les conflits nombreux pour des questions d'argent : celui qui a trouvé le café-restaurant où jouer doit-il être plus payé que les autres? Certains intermittents attaquent leurs collègues en Prud'homme. Parmi les plus stables de ces précaires, une grande partie a constitué un réseau de lieux et de fans dans une métropole, 
ce qui permet d'assurer une régularité des prestations. Les fans suivent les différents lieux, une partie du répertoire leur est réservée, ainsi est-on catégorisé par un style, blues, jazz manouche, latino... Ensuite, il faut raisonner par " projet ", tarte à la crème des agences pour l'emploi, des écoles d'éducateurs, des universités, des industries classiques. Ainsi, les prestations peuvent-elles provenir d'un club de cinéma, de danseurs, de choristes, de peintres, de radios . Il faut soigner ses fans, entretenir le maximum de relations dans tous les domaines et surtout travailler intensément la technique de l'instrument ou de la voix, comme on le fait dans les challenges américains. Quelques-uns se sont établis aux USA, Canada, Grande Bretagne, Berlin pour tenter leur chance. Connaître les organisateurs de festival et leurs organisations permet parfois d'entamer des tournées internationales, d'intégrer un orchestre du show-business, mais il faut une solide formation de lecteur de partitions, être sociable.

Daniel Givonne, un passionné, splendide guitariste et compositeur, résume son activité : d'une famille mélomane, il arrête l'école à 14 ans, va à Paris où il joue du Musette et du Django. «C'était pas la mode... Ensuite j'ai eu une grosse révélation en 1973 (...) en 1994, ma femme de l'époque (une jolie blonde ) faisait les pompes et les manouches ont été enthousiastes ». Il faut faire ses preuves d'enregistrement et de production, puis des enregistrements chez les Majors, Pathé, Barclay, Polydor. Comme tous les musiciens, il auto-produit des $\mathrm{CD}$ de chaque série des nouvelles compositions qu'il vend à la fin des concerts, « ça ne coûte pas cher, 2000 Euros pour un enregistrement. Mais on n'a pas pas les conditions de son des gens des studios.». Il travaille le son de ses guitares, ses compositions l'éloignent des standards manouches et il est à mon avis l'un des dix premiers guitaristes en France. "On n'était pas assez commercial, c'était le constat à l'époque, on ne les a pas intéressés, contrairement aux USA ou au Canada. Aux USA, le management recherche, en France, on ne voit pas ça. J'ai un pote, il a été aidé par les banques en France, ça n'a pas fonctionné (...) Ici l'industrie du disque, il n'y a pas de culture, ils sont saturés d'artistes». Il écoute beaucoup les nouveautés d'Espagne, de Grande Bretagne, d'Allemagne, va jouer en Asie.

«On ne fait pas de victimisation, on est responsable, nous aussi. D'ailleurs, tu n'as pas besoin d'être un super homme d'affaire, tu joues, on t'entend. Le bouche à oreilles, « tiens, toi, tu viens par-là ». Le système parisien, c'est pas du tout mon truc, il faut faire ses preuves tout le temps. La moindre fatigue... C'est beaucoup plus difficile, c'est un combat. Faire la lutte tout le temps, c'est pas intéressant ». Il critique les majors comme Drefus, le blues trop facile, la concentration des bons musiciens à Paris « tout le monde va là-bas » et rend hommage à des guitaristes comme Sylvain Luc: «le summum. Feeling, technique, humanité. Il existe de grands talents en France, mais le travail à faire, c'est la promo. ». La grosse part du travail, c'est la publicité, qui ne se marchande pas, il faut tout faire, « on est sur la bride de la Com, avec les industries, il faut prendre ses distances, elles cherchent des compils, nous, le but du jeu, c'est de tourner, il faut faire des photos tout le temps ». Après chaque tournée, il faut enregistrer sous un mois, payer des gens pour filmer les concerts et en même temps composer pour le prochain disque, «vendre les CD, c'est notre cagnotte, (...) pour le prochain, c'est combien, pa, pa pa, pa, je vais dire à quelqu'un.... Tout est comme ça. ». Les vidéos sur You Tube ne rapportent rien, mais ça fait connaître.

Certains couples qui ne se revendiquent pas musiciens doublent leur salaire mensuel en jouant de la musette avec accordéon et synthétiseur, plus les chants le samedi-dimanche, lui était professionnel à 14 ans le samedi soir « tu me ramènes une paire d'oursins (soit un 
smic) » disait sa mère. Mais les musiciens avaient peur des bagarres dans les bals pendant les années 80. Payé à la soirée, il continue une trajectoire classique dans le milieu, chef d'orchestre «je ne gagnais rien, mais c'était très bien", signe des contrats, classe les formations et les salles des fêtes, les Comités d'Entreprise lui font confiance, "ils recrutaient direct pour l'année suivante. Il y avait des musiciens incroyables, c'est un très bon coin pour apprendre, mais après, j'en avais marre, ce n'était pas la vie que je voulais». Il devient musicien indépendant. "On peut être dans un super festival, le lendemain on fait de l'animation ou jouer dans un troquet pour 100 Euros ». Il se plaint de l'académisme parisien : «On ne rentrait pas dans le moule, le critère des mecs, c'était de faire comme Police ou Golmann, commencer une chanson par le couplet, c'était interdit. Mais il y a d'autres métiers, être journaliste, tu fais ce que tu veux: Trust, Johnny Halliday, Téléphone, Louise Attack, il faut être pris en main. Des copains musiciens de studio ont monté Pow How, ça a marché deux ans, ils avaient une banane terrible, et finalement l'un d'entre eux s'est acheté une guitare Gretch (50 000 Euros), c'était son rêve, après, ils ont arrêté ». Avec la suppression des subventions municipales envers les radios libres, la notoriété passe désormais par des émissions de télévision comme the voice

"Comment reconnaître un mec à sa juste valeur? Dans le show-bizz, il a des mecs à dégager. C'est comme pour Pow How, je m'achète une guitare parce que je ne vois plus devant, les producteurs ne suivent plus, comme pour Jacques Brel. Ca ne tiendra pas. On n'est plus dans le moule. (...) Chez les producteurs, ce n'est jamais le même mec, il faut faire carrière, ramener le maximum d'argent générer de l'argent, c'est hallucinant, en tant qu'intermittent, on gagne 600 euros par mois, plus les cotisations, le Hellfest (à côté de Clisson, 44), c'est 80 camions et 1,5 millions d'Euros pour un groupe (...) L'empire du disque, c'est une commande. Ils se connaissent tous, ils détiennent quelque chose, il y a une centaine de personnes à Paris et ils jugent : ça ne se vendra pas. Pourtant Magma, Ange, Higelin, c'était gigantesque, ils auraient mérité».

Il connait parfaitement les trajectoires sociales de Tchavolo Schmidt, " un grand maître ", sa compagne s'occupe des relations financières et avec les avocats car les plagiats de musique manouche déposée sont nombreux; contrarié ce jour-là, il n'a rien voulu me dire, « ils ne savent pas jouer ». Romane, Bireli Lagrène et ses oppositions familiales, et nos idoles, Paco de Lucia "poussé par son père ", Tomatito et bien sûr Dorado Schmidt, un homme de coeur, nous avons évoqué ces artistes avec beaucoup de sensibilité. Les cercles musiciens manouches sont très bien organisés d'un point de vue juridique, technique, économique et il est facile, à l'occasion, de s'y faire adopter lors de leurs rassemblements.

Vers les années 2000, Daniel est « convoqué » par le directeur de l'organisation du festival des Rendez-vous de l'Erdre à Nantes (3 jours fin août), « tu as un bon état d'esprit », et il entame une tournée au Japon, puis au Népal. Chaque année, il présente une nouvelle formation à Nantes et de nouvelles compositions. Il travaille « en famille » à la maison, est de plus en plus invité en Europe, les femmes gèrent l'argent comme dans beaucoup de couples populaires. " $\mathrm{j}$ 'en avais marre d'être catalogué comme manouche. Au Japon, en Allemagne, on a commencé à se décoincer de tous les côtés. Avec la diffusion des mails, on a vachement tourné, un événement en crée un autre, en international, il y a peu de concurrence, il faut être contacté directement. »

Comme beaucoup de musiciens, il a été intermittent, puis arrêté, puis revenu, « ça n'est plus ce que c'était, pas plus payé pour faire une animation qu'il y a dix ans. On a des tarifs 
réduits, 3500, 5000 Francs, mon frère gagnait 35000 Francs par mois en 1988. Maintenant, Goldmann et la variété imposent leurs tarifs, pour un mec connu qui remplit un Zénith, c'est 1500 Euros par soirée. Romane ou Angelo Debard peuvent avoir 6000, Lavilliers ou Catherine Ringer un peu plus parce qu'ils ont des producteurs ».

Michel Pérez a suivi une trajectoire à peu près identique, instituteur, il a suivi les cours d'une école à New York où il rencontre des "gros calibres », puis monte un groupe de jazz-rock à Grenoble, aidé par la Maison de la Culture, « il fallait créer de la musique, on avait des contrats, c'était un période faste (à la fin des années 70) ». Immigré espagnol, ses choix musicaux ne viennent pas de la famille, l'important était d'avoir un travail, il faut apprendre à rédiger des dossiers d'aide pour les subventions, «se dépatouiller avec les papiers ». Il joue avec son groupe en première partie de Miles Davis au théâtre de Vienne en 1984, je me souviens avec un copain guitariste que ça nous amusait de voir qu'ils avaient le trac, alors qu'on l'avait nous aussi lorsqu'on allait jouer à la Fête de l'Huma. Il est timide, pas du tout le genre «bête de scène » et il a choisi de vivre tranquillement à Paris, où il joue chaque semaine, après avoir écrit la musique d'un film de Tavernier.

«Le road de Miles, les musiciens viennent directement, sans préparation, on n'a même pas le temps de faire les balances pour jouer une heure, je ne le referai pas. On gagne 100 pour un spectacle qui coûte 10 000, j'ai rencontré John Scofield (guitariste), un mec gentil, et Al. Foster (batteur), rassemblé devant un frigo sous une tente, on a bu du champagne. Le lendemain, on devait jouer à Nice, il y avait grève des trains, on n'avait pas de voiture ». Mais la disproportion entre les cachets ne le choque pas vraiment. Dominique Di Piazza, son bassiste, avait été remarqué par le guitariste international Mc Laughing qui jouait avec Miles Davis : « ils jouaient en trio, en Australie, dans les télévisions, c'est une musique complexe, il faut savoir lire, savoir décharger son agressivité. Les cachets sont différents aux USA, en Grande Bretagne, en Amérique latine où on n'est pas déclaré. Dominique gagnait 400 euros quand le guitariste touchait 15000 ». Michel a embauché deux ou trois agents pour chercher des contrats et organiser une tournée en Corée du Sud où il rencontre à nouveau des artistes internationaux, il donne des cours, joue avec des orchestres symphoniques, «ça a fait boule de neige. J'étais avec Hancock et Shorter, Tavernier s'est renseigné et on a enregistré « Autour de minuit » pour son film en 1986 ».

41 Il refuse d'avoir recours à des " tourneurs», des agents qui organisent grâce à leur portefeuille de relations des tournées plus ou moins réussies sur le territoire national, européen ou international (j'ai été témoin de grosses escroqueries) moyennant ponction sur le chiffre d'affaire. "Il faut être affilié à une compagnie de disque importante, j'ai pas réussi à rentrer dans ce réseau-là, il faut être scénique, mais je préfère la musique intimiste. Je ne démarche pas ».

Parmi les musiciens merveilleux, Michel n'est pas le seul à refuser le vedettariat. Alain Jean Marie, du même âge, 72 ans également, témoigne ainsi : Il n'a pas fait d'école, a appris la musique « avec les oreilles » depuis son enfance dans les Antilles, joue dans les bals puis à 22 ans à Montréal, via le Pavillon Créole financé par le Conseil Général, s'autoproduit, a des entretiens dans les magazines, rencontre d'autres musiciens Martiniquais, "à l'époque, on faisait du rock ", monte un groupe de fusion, puis après une tournée en Haïti et au Maroc , arrive à Paris où il est embauché comme organiste à la Cigale. Vers 1973/74, entre les hôtels et son cachet, il lui reste 5 Francs par jour, enregistre avec des Américains connus pour des tournées européennes, " je suis venu du jazz, du Bop. Chaque musicien a un agent, ce sont des guides qui t'engagent dans le business. Le patron, c'est le chef d'orchestre qui me paie, c'est le leader du groupe, il a l'affaire. Depuis les 
années 2000, on n'a plus d'engagement sur une semaine, c'est 2 ou 3 jours, une seule journée, c'est fréquent, il faut se débrouiller avec les hôtels ».

Son ami, François Ripoche, persiste lui aussi dans le jazz, professeur dans les écoles, membres de plusieurs formations, réalisant des vidéos avec un artiste connu et avec lequel il s'en va pendant des tournées, jouant du saxophone en public pour des films dont on a coupé le son, ou régulièrement dans un café où il excelle. «Je joue partout : le public de jazz n'est pas nombreux, il faut trouver un peu de finances». Le monde est petit, les musiciens se connaissent tous et je n'ai pas l'impression de jalousies ou d'agressivité ou de frustrations comme il est notable dans les administrations. Les références en musique classique ou en pop musique sont communes, il faut trouver des affaires, embaucher un prospecteur dès qu'on a un chiffre d'affaire suffisant, surveiller les sites internets. Parfois, après une longue période d'absence, les musiciens remontent sur scène avec un nouveau groupe. "Le monde de la musique est tout petit. Il faut jouer de tous les instruments, taper à toutes le portes, avoir un bon carnet d'adresse, avec les tourneurs, les producteurs, les télévisions, radios, il faut s'occuper de tout ».

«Les nouveaux artistes sont mieux cachés, mieux encadrés. Beaucoup se sont fait avoir parce qu'ils n'avaient pas payé leurs impôts, la nouvelle génération est plus gestionnaire, tant mieux. C'est un peu comme au football, il ne faut pas se faire entuber en essayant d'avoir des sons à la frite comme Goldmann. J'ai été intermittent pendant 40 ans nous dit Ismet Mered, je me suis retiré, maintenant, je suis prestataire de service. J'aime travailler, il y a plein de gros bosseurs. (...) J'étais chef d'orchestre, je ne cherche pas la gloire, j'étais bien payé. Le système a changé avec la Musique Assistée par Ordinateur pendant les années 80, mais les meilleurs techniciens subsistent, les vedettes et les producteurs les recherchent, il faut avoir l'intelligence de comprendre, avoir l'esprit ouvert, voyager. Le musicien a toujours tendance à s'enfermer chez lui. (Il énumère les vedettes avec lesquelles il a joué). Une fois que c'est fini, c'est fini, on ne se voit plus. On fait semblant d'être copain, t'es sympa, mais il faut que ça passe, tu n'es pas redevable. (...) L'esprit anglo-saxon, ce sont des bosseurs, il faut être reconnu sur le travail ; le latin, c'est plus de la frime, il ne pense qu'à sa gueule et il donne des leçons. En France, la télé, ça n'est même pas payé ».

\section{Des modes de vie homogènes}

La plupart des musiciens rencontrés ont finalement des modes de vie relativement homogènes, malgré la diversité des styles et des spécialisations. Nombreux sont ceux qui évitent de s'inscrire comme intermittents, où il faut assurer 507 heures de travail déclarées sur 12 mois pour être auto-entrepreneur par exemple; «c'est trop contraignant, il faut tout le temps donner des dates ». Les charges de cours dans les écoles et associations permettent également d'assurer une base de revenu autre que la scène. La double ou triple activité étant la règle, ils ont eu fréquemment des emplois dans les administrations sanitaires (psychologues, musico-thérapeutes, infirmiers) ou scolaires, enseignants, ou parfois à leur compte (pizzaïolo, viticulteur). Les voyages et les rencontres en Amérique, Afrique, Asie avec des «bêtes de scène » ou des vedettes internationales jouent un grand rôle dans les mémoires et la constitution de formations pluri-nationales est très fréquente. L'embauche dans des entreprises de création d'événement, où les cachets sont supérieurs à ceux de la scène musicale est recherchée. 
On se souvient d'avoir vécu des périodes financièrement fastes et d'autres où on ne gagnait rien, d'avoir joué tous les styles, autrefois dans les bals et les boites de nuits avant la prise de pouvoir financier par les DJ, d'avoir été catapulté dans des tournées par de mystérieux producteurs. Il a fallu apprendre la scène, les techniques d'enregistrement lorsqu'on est passé des magnétophones à cassettes, des mythiques Revox à bande, puis à l'informatique, s'être fait escroquer par « des pseudo-managers bien véreux qui financent des albums et piquent dans la caisse, qui paient avec des chèques en bois ". "Je me suis fait enfler par les droits d'auteur, pour la fille d'un musicien qui était chanteuse, j'avais fait tous les arrangements, j'ai bossé comme un malade pour rien du tout ».

Lorsque la réputation et les réseaux fonctionnent bien et que l'on est «signé par une maison de disque ", encore faut-il être attentif à la qualité de son choisi, car les techniciens peuvent imposer leurs automatismes selon des choix numériques et produire une musique froide. « il y a un problème de professionnalisation dans les studios, ce sont des automates ». Lorsque des artistes sont signés par des majors du disque (comme EMI), certains refusent de laisser la moitié de leur chiffre d'affaire à la firme. Toute une série d'intermédiaires se sont donc créés autour des artistes, en général venus des milieux universitaires, dont ils entretiennent le narcissisme, « ce sont des parasites qui se mettent en valeur, qui magouillent avec les associations et les subventions, qui embauchent des étudiants pour rien du tout $»$.

Dans les métropoles, la mise en concurrence des inventions des œuvres authentiques et du Patrimoine, des circuits de financements et de tournées comme ceux de la Fédélima (un réseau national d'agences départementales subventionnées pour trouver des concerts), du Centre national de la chanson de variété et du jazz (CNV), des scènes de la musique actuelle (SMAC), des maisons de la Culture et des municipalités, les associations de cafés-restaurants qui regroupent parfois plus de 400 établissements, ont brouillé les trajectoires classiques ou ordinaires des musiciens. La multiplicité des intermédiaires diplômés et les changements techniques ont bouleversé l'organisation des réseaux d'entreprises. "J'ai monté une société de production, j'ai été tourneur, organisateur, c'était la belle époque, il y a 20 ans, on pouvait gagner 800 Francs par jours. Il y avait du boulot pour des orchestres de 15 personnes. Maintenant, le DJ arrive, tout est enregistré, c'est lamentable, et il fait des thés dansants quatre fois par semaine dans les maisons de retraite ».

Les arts du cirque et du théâtre, comme tous ceux du spectacle vivant où présence scénique et corporelle, musique, valorisation des décors, costumes, textes et espaces, ont suivi le même chemin; " on avait vingt instruments et cinq personnes, on tournait au Canada, en Belgique, en France, 70 concerts par an, des intermittents, des professionnels et lorsqu'il a y eu les grèves à Avignon, on a perdu 12000 Euros et revendre les dates était impossible. (...) Maintenant on est trois. On a tous collaboré dans le théâtre, on joue la comédie, on envoie nos CD par la poste et on téléphone. (...) Le marché a changé depuis 15 ans, on ne cherche pas la notoriété, il n'y a plus de tourneur, mais une baisse du pouvoir d'achat. Dans le public ou dans le privé, on vendait un spectacle 7000 Euros, maintenant, c'est 1500, mais on a toujours 5 ou 6 projets ». Même le théâtre conventionnel où se produisent de grandes vedettes a recours à des bénévoles, flattés par la notoriété de tel ou tel artiste; en général ils ont joués dans de multiples compagnies. Les plus prudents des bénévoles ont passé des formations pour devenir directeur d'une maison des jeunes où il faut gérer à la fois les spectacles, le public et les relations avec les équipes municipales, d'autres sont devenus techniciens son et lumière. La différence avec les musiciens est que 
les équipes sont logées dans le même appartement lors des tournées et que certains comédiens connus peuvent imposer leur propre style au metteur en scène, comme au cinéma.

A la différence de la plupart des enseignements universitaires et de ceux qui en sortent, le niveau technique ne cesse d'augmenter en musique. A chacun de l'utiliser, selon son libre choix, mais on peut aussi n'en rien faire et gérer les compétences des autres. On retiendra que dans les imaginaires français, l'Administration prime sur toute autre initiative, qu'elle juge, ce qui fait peut-être notre charme européen et produit pas mal de gaudrioles. Mais la passion des musiciens rencontrés nous guide vers d'autres imaginaires, ceux des voyages, des tournées, des rencontres, des illusions momentanées que l'on tente sans cesse de faire perdurer. Les artifices et la société du spectacle ont encore un futur radieux, et des découvertes fabuleuses, un peu comme cette péninsule, que promettait Don Quichotte à son fidèle aide de camps Sancho Panza.

Lévy Strauss Claude (1979), «La voie des masques», Michaud Yves (2012), « Ibiza mon amour. Enquête sur l'industrialisation du plaisir », ed NIL ed. Plon Menger

Pierre Michel, (2002), « Portrait de l'artiste en travailleur », ed.Seuil

\section{BIBLIOGRAPHIE}

Bruno Lefebvre « Le Mal », juin 2007, conférence Lestamp, Médiathèque de Nantes.

Lefebvre Bruno (2015), « Les tribulations d'une innovation musicale : l'exemple des « Fauteuils Branchés » in Volume! $\mathrm{N}^{\circ} 11-2$

\section{NOTES}

1. - conférence Lestamp, «Le Mal», juin 2007, Médiathèque de Nantes, Bruno Lefebvre, «La sociologie a de l'influence sur la société : Gustave Le Bon, psychologies des foules et son élève Herrmann Goëring, journal quotidien 1925/45 »

2. Boltanski Luc, Chiapello Eve (1999), « Le nouvel esprit du capitalisme, ed . Gallimard

3. Menger Pierre Michel, (2002), « Portrait de l'artiste en travailleur », ed.Seuil

4. Guibert Jérôme (2006), «La production de la culture. Le cas des musiques amplifiées en France », ed. Mélanie Seteun

5. Lefebvre Bruno (2015), «Les tribulations d'une innovation musicale : l'exemple des « Fauteuils Branchés » in Volume ! N¹1-2, ed.Mélanie Seteun

6. Michaud Yves (2012), « Ibiza mon amour. Enquête sur l'industrialisation du plaisir », ed NIL

7. Lévy Strauss Claude (1979), « La voie des masques », ed. Plon

8. Lefebvre Bruno (1992), «Les dynamiques d'un espace professionnel », micro-film, thèse, bibliothèques universitaires 
INDEX

Mots-clés : Industrie de la culture, industrie du disque, artistes ventouses, techniques de production musicale, DIY

\section{AUTEUR}

\section{BRUNO LEFEBVRE}

Professeur des Universités, Nantes, membre du LISE-CNRS 